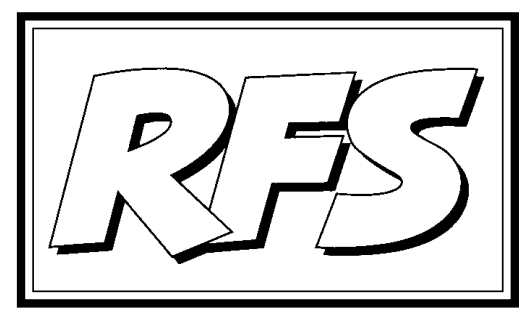

Revista de Fomento Social, 57 (2002), 683-710

\title{
Razones para implantar un sistema de evaluación en las ONGD y sus consecuencias
}

José María LARRÚ

\section{Introducción}

¿Es eficaz la ayuda al desarrollo que ejecutan las ONGD españolas? ¿Sirve realmente para algo, sobre todo para reducir la pobreza en los países donde se realiza la ayuda? ¿Llega esta ayuda a los más pobres y necesitados? ¿Puede el donante fiarse realmente del destino de los fondos canalizados a través de las ONGD? ¿Cómo se controlan esos fondos? ¿Son las ONGD organizaciones de aprendizaje o reproducen sin más los proyectos sometiendo la información que les llega de sus contrapartes del Sur a la disciplina del formato del Marco Lógico exigido por las financiadoras? ¿Cuál es su valor añadido en la cadena de la cooperación al desarrollo?

* Profesor Adjunto del Departamento de Economía General. Facultad de Ciencias Económicas y Empresariales. Universidad San Pablo-CEU.

1 Esta cadena es la forma de expresar la sucesión de actores que intervienen en una acción de 
Estas o similares preguntas son de indudable interés tanto científico (¿cuál es la verdad sobre la ayuda de las ONGD?), como político (el control de las subvenciones) o social (las preguntas críticas de una sociedad civil educada en el problema del desarrollo). Y muchas de las respuestas pueden lograrse instaurando en la ONGD un buen sistema de evaluación de sus acciones.

En este artículo se pretende motivar a dichas Organizaciones a pensar en el valor de decidirse a aplicar un programa de evaluaciones útiles que las conviertan en lo que Edwards (1997) ha denominado "organizaciones de aprendizaje". Las ONGD españolas gestionan directa o indirectamente un $35 \%$ de la Ayuda Oficial al Desarrollo (AOD) española (Tejada, 2002:122) ${ }^{2}$ superando ya los 540 millones de euros anuales ${ }^{3}$ y desde el año 2001 pueden obtener financiación estatal a través de tres instrumentos básicos: convocatoria de proyectos, de programas y de estrategias ${ }^{4}$. Este nuevo acuerdo de cofinanciación ha incluido una partida del 1,5\% del presupuesto para evaluación, especialmente en los casos de programas y estrategias, en las que se deben rendir cuentas anuales mediante informes de evaluación cuyo contenido y formato preciso aún no se han detallado por parte de la Administración. Pero en cualquier caso, el refuerzo de la actividad evaluadora en todos los instrumentos de cooperación al desarrollo ha sido una de las recomendaciones del CAD en su pasado examen: "Se insta al MAE [Ministerio de Asuntos Exteriores] para que siga haciendo lo posible por reforzar el control y la evaluación en el sistema de ayuda español, en particular por lo que respecta a

ayuda al desarrollo: cómo, normalmente, la financiadora dona el dinero a la ONGD del Norte tras superar el proceso de selección de los proyectos presentados en papel, la ONGD del Norte traspasa los fondos a la ONG del Sur o contraparte local, y cómo ésta organiza la ejecución de la acción sobreel terreno, contratando a las empresas y personal necesario para lograr el objetivo proyectado. Cuanto más larga sea esta cadena, mayores son los costes de transacción y más ineficiente puede ser la ayuda al desarrollo, como ha expuesto el actual director de la Oficina de Planificación y Evaluación de la Cooperación Española. TEJAdA (2002).

2 Según el reciente examen del Comité de Ayuda al Desarrollo (CAD) de la OCDE a la cooperación española (CAD, 2002) "junto con las donaciones de la AECl, aproximadamente el $29 \%$ de la AOD bilateral, o el $39 \%$ de las donaciones, se canalizaron a través de las ONG entre 1997 y 2000".

3 Unos 90.000 millones de pesetas. La cifra corresponde al total de recursos del ejercicio de 1999 último año del que se dispone el dato de la Coordinadora de ONGD en su Directorio de 2000. Los pasados ejercicios no se publicó esta información por falta de medios de la propia Coordinadora creando un vacío lamentable que esperemos se resuelva lo antes posible. Para el análisis detallado de los últimos datos disponible veáse LarRú (2001a).

4 Para mayor detalle véase el artículo en esta misma revista, LARRú (2001b). 
los créditos FAD, la cooperación descentralizada y las ONG, y para que destine más recursos a tal efecto. Es imprescindible organizar de forma más sistemática la difusión de las lecciones extraídas". CAD (2002: 56).

El trabajo está basado en un estudio más amplio que vio la luz en forma de tesis doctoral en la Universidad San Pablo-CEU (Larrú 2000). Allí se ofrecía un diagnóstico sobre el sector de las ONGD españolas en materia de evaluación que reflejaba que muy pocas entidades estaban llevando a cabo alguna misión evaluadora, y cuando ésta se hacía, normalmente se externalizaba a través de una consultora (con lo que su coste era elevado) y estaba motivada por algún problema grave acaecido en el proyecto evaluado. Sobre este diagnóstico, se ofrecían diversas metodologías para evaluar los proyectos, basadas en la literatura tradicional que principalmente ha generado la pedagogía norteamericana, y se proponían los cambios que las ONGD debían llevar a cabo para implantar un sistema evaluativo eficaz. Desde entonces la situación ha cambiado poco. La mayoría de las ONGD españolas siguen expresando su deseo de evaluar y aprender y reconocen el no hacerlo como una laguna en su gestión, pero no se deciden a poner remedio a esta carencia. Es por esto que el artículo quiere volver a insistir sobre la importancia del tema, dejando para otra ocasión los problemas de metodologías más o menos adecuadas a la realidad de los proyectos de desarrollo ${ }^{5}$.

El trabajo se estructura de la siguiente manera. En primer lugar se exponen las razones posibles por las que hasta ahora las evaluaciones de impacto en las ONGD no sean más que excepcionales y se ofrecen razones positivas para adoptar la decisión de la instauración de un sistema de calidad total basado en los aprendizajes obtenidos a raíz de la ejecución de evaluaciones en todas

5 De todas formas, las cuestiones generales sobre la materia pueden encontrarse bien expuestas en las Metodologías de Evaluación de la Cooperación española editadas por el MAE (1998; 2001). GonzÁLEZ (2000) también ha introducido de forma general el tema y para una visión más concreta de los paradigmas evaluativos pueden consultarse los manuales de WEISS (1998) y Rossi y Freeman (1989) de corte más positivo o clásico-ortodoxo-cuantitativo, GuBa y LINCOLN (1989) para el paradigma naturalista o más cualitativo; las aportaciones de PATTON (1997) insistiendo en que la evaluación debe ser útil y puesta en práctica; o de CHAMBers (1997) que es el principal divulgador de los enfoques participativos. Para la necesidad de combinar instrumentos cuantitativos y cualitativos y superar los enfoques tradicionales en las evaluaciones de la pobreza véanse SaLmen $(1995,1998)$ у Rовв (1999) que describen el proceso del Banco Mundial y CARVALHo y WhITE (1997) y WhITE (2001) que ponen el énfasis en la complementariedad de los enfoques cuantitativos y cualitativos. Una referencia obligada en el tratamiento de la evaluación en la cooperación al desarrollo es el conjunto de artículos del número monográfico de la Revista Española de Desarrollo y Cooperación IUDC-UCM (1999). 
sus acciones. En segundo lugar se explican algunas de las consecuencias que tendrá para la ONGD realizar esta opción. Se consideran los cambios que el ejercicio sistemático de evaluar tiene sobre la identidad de la ONGD, así como los cambios organizativos, presupuestarios y sobre los recursos humanos de la organización.

\section{La decisión de evaluar}

Aunque parezca una obviedad, sin la seria determinación y convicción de la utilidad y necesidad de la evaluación de las acciones que realiza la ONGD, todos los esfuerzos que se orienten en esta actividad no tendrán éxito.

Cabe la posibilidad de declarar abiertamente el deseo e interés por hacer evaluaciones, pero sin un empeño firme y constante, serán vanos, máxime teniendo en cuenta las dificultades y complejidades inherentes a la implantación eficiente del ciclo de la evaluación. En otro trabajo (Larrú 2000) dimos cuenta de cómo en la muestra de las ONGD españolas que acumulaban el $80 \%$ del gasto en proyectos, el $15 \%$ de las organizaciones confesaban abiertamente no haber hecho nunca un ejercicio de evaluación de sus acciones, el $85 \%$ restante confesaba evaluar, pero entendiendo ésta como la entrega del informe final exigido por la financiadora. Únicamente al guna ONGD demostró haber encargado una evaluación intermedia en profundidad a un grupo especializado cuando el proyecto se complicó en exceso.

Antes de exponer las razones que justifican la implantación de un sistema de evaluación podemos considerar algunas de las posibles alegaciones para no haberlo hecho todavía.

En un primer estudio, Riddell (1990) señaló algunas de ellas como la ausencia de presiones o demandas externas para hacerlo, la sobrecarga de trabajo del personal interno de la ONGD, el que cada proyecto sea único y haga imposible toda validez externa, los objetivos tan generalistas de los proyectos y el que suelan cambiar a lo largo de su ejecución, la falta de examen previo, línea de datos de base y grupos de control, o el temor a reconocer el propio fracaso. El propio Riddell (1998) sistematiza las principales razones para no evaluar en estas seis:

- Un primer argumento, al que podríamos denominar «necio»o autosuficiente, es el que considera la evaluación como una moda pasajera, algo economicista y que no aporta nada valioso al trabajo de la organización. Por su simpleza, no merece más comentario, aunque 
hay que confesar que hace diez años, pocas ONGD expresaban abiertamente la necesidad de evaluar sus proyectos ${ }^{6}$. Esto, por fortuna, ya ha cambiado radicalmente.

- Una segunda postura es la «paternalista». Esta argumenta que las ONGD existen como respuesta al «grito de los pobres» y que, mientras exista este grito, ellas se sienten legitimadas para actuar, sin necesitar evaluaciones que gastan preciosos fondos en detectar impactos o eficacias. La necesidad de la gente es «muy real», y la ayuda es -por sí mismasuficiente base legitimadora de sus actividades.

- La tercera postura ya es más audaz. Podríamos llamarla la «unívoca». Para esta postura las intervenciones y proyectos de las ONGD son únicos e irrepetibles, por lo que las lecciones que puedan extraerse de uno, no son extrapolables al resto. No hay posibilidad de validez externa. Los aprendizajes de cada proyecto, están subsumidos en él mismo, y los beneficiarios conocen de sobra lo que el proyecto les ha aportado y lo que no. Esto es lo que realmente importa: si una población tiene ahora agua potable y antes no, si dispone de una escuela o no. No hace falta «evaluar»artificialmentelas acciones de desarrollo porquese autolegitiman y cada proyecto es único en el espacio y el tiempo. Al no poderse volver a repetir esas mismas condiciones no tiene sentido evaluar, pues ninguna acción es reproducible exactamente como se formuló en una ocasión. Así, no se pueden establecer relaciones biunívocas entre proyectos y la validez externa entre ellos es cero. Cada uno es único e irrepetible.

- El cuarto motivo es de orden «ógico». Se basa en la dificultad o imposibilidad de separar causas y consecuencias dentro del universo relacional del proyecto. Hay efectos que inevitablemente ocurren junto al proyecto, pero que no deberíamos atribuir a él, ni podemos separarlo de él. No podemos «diseccionar» artificialmente la realidad, sino que debemos considerarla globalmente. El elemento "proyecto de desarroIlo", es inseparable del conjunto "realidad económica de la zona" y por tanto, al evaluar sólo el proyecto, dicha evaluación estará tan sesgada que no resultará relevante. En el fondo se plantea la conveniencia de realizar evaluaciones que superen la dimensión del proyecto, y se

6 Por ejemplo en Edwards y Hulme (1996:260), se mantiene que: «la mayoría de las ONGs no se sienten afectadas de ningún modo por las cuestiones de rendición de cuentas (accountability)». 
estaría abogando por los enfoques más generales como las evaluaciones temáticas, sectoriales o geográficamente amplias (región o país).

Sin negar la parte de verdad que tienen estos dos argumentos, creemos que lo que falla es la consecuencia. Evidentemente es muy complejo evaluar y tiene una dimensión subjetiva como subjetiva es la realidad. Pero la dificultad no debe llevar a la inacción. Por poner un paralelismo, investigar es costoso, exige esfuerzo y nunca se puede dar por acabado un buen tema de investigación. No siempre se cuenta con los medios suficientes y nadie garantiza que de una investigación básica se obtenga una innovación productiva que rentabilice el gasto ejecutado. Pero todas estas dificultades no generan la consecuencia de la completa renuncia a investigar. Se hace lo que razonablemente se puede, como mejor se puede. Pero algo se hace.

- En quinto lugar, nos encontramos con el argumento «procesual». La dificultad que se plantea aquí a la evaluación es la de considerar que el desarrollo debe ser visto como un proceso continuo de largo plazo, y no como un conjunto desarticulado de proyectos que a menudo pueden presentar objetivos artificiales. Para los más extremistas de esta postura, la misma evaluación no es posible, ya que los impactos son tan globales, en la actual coyuntura internacional, que no pueden detectarse con rigor. Otros, menos extremistas, reconocen la validez de los modernos métodos y herramientas de evaluación, pero consideran que están basados en unos "a prioris" tan artificiales que los invalidan en sus propios resultados.

- La última postura o reticencia frente a las evaluaciones, puede ser la más común. Es el motivo de vivir el proceso evaluativo como una «amenaza» o control externo. La presión que pueden sufrir los implicados en la evaluación (por ejemplo si saben que de su información dependerá la continuidad o no de los fondos enviados por el donante), puede ser tan grande que sesgue los resultados y se describan impactos que realmente no existan. El razonamiento -consciente o no- funcionaría así: "cuantos más impactos encontremos, más fondos seguiremos recibiendo. Luego hagamos un informe «estrella» sobre el proyecto". Piénsese su relevancia para ONGD medianas y pequeñas que sobreviven gracias a las subvenciones que obtienen de organismos cofinanciadores. Una mala evaluación podría ser determinante en su futuro así que, o no la harán, o la sesgarán positivamente hasta invalidar su credibilidad. 
A éstas razones podrían añadirse las consideraciones de coste y alto precio de las evaluaciones. Al percibir la evaluación como un gasto en vez de como una inversión, no se aprecian los beneficios de coste de oportunidad que supone no hacer evaluaciones ${ }^{7}$.

Pueden aparecer consideraciones de pequeña escala de la organización, de sobrecarga de trabajo, de falta de presupuesto, o de cualquier otra índole. Seguramente aquí sea aplicable el aserto de que no se demanda lo que no se ha probado o no se conoce por experiencia previa. La ignorancia culpable y la resistencia acomodada a la inercia en la forma actual de trabajar de las organizaciones, serán obstáculos no fáciles de vencer por aquellas entidades que estén dispuestas a instaurar de forma eficiente un ciclo sistemático de evaluación de sus proyectos y programas ${ }^{8}$.

Pero no sólo caben posturas negativas contrarias a realizar evaluaciones sino que también pueden darse razones poco legítimas para encargarlas. García Ramos considera las siguientes ${ }^{9}$ :

- Aplazar una decisión mediante el encargo de un estudio evaluativo que requerirá más tiempo.

- Eludir responsabilidades ante decisiones ya tomadas, pero que se desea revestir del "ropaje legitimador" de la evaluación.

- Encargar la evaluación con fines de marketing y propaganda, como medio de autoproclamar las grandezas de la organización. Para ello no se duda en seleccionar para la evaluación únicamente aquellos rasgos del trabajo que tienen un buen aspecto superficial, o encubriendo los fracasos del proyecto evitando toda estimación objetiva y sin ninguna difusión ni transparencia en sus resultados.

- Cumplir los requisitos obligados de una convocatoria de financiación

7 Pensemos por ejemplo, en el ahorro asociado a no ejecutar proyectos fallidos o sistemáticamente mal diseñados, por no detectar los errores gracias a una evaluación. 0 las decisiones tomadas sobre impresiones superficiales o conjeturas en vez de lograr certeza por las conclusiones de un ejercicio evaluativo.

8 Las analogías con las resistencias dentro de las organizaciones empresariales a generalizar los procesos de calidad total en sus procedimientos y sistemas, pueden ser una comparación pertinente, a la que habría que añadir la falta de presión por el lucro y beneficio sobre la que descansa la actividad de la ONGD.

9 García Ramos (1992:463-464), siguiendo a Weiss (1972) en su versión castellana (1975). 
externa, convirtiendo la evaluación en un ritual necesario que se manipulará para convencer o "aplacar" al financiador, pero carente de una utilidad real.

Esta última razón ilegítima nos parece especialmente importante para nuestro contexto de evaluación de proyectos y programas de ONGD, financiados en un importante porcentaje por organismos públicos que demandan una rendición de cuentas.

No hay que olvidar que en toda evaluación se unen motivaciones políticas (toma de decisiones) con análisis de efectos o resultados económicos y sociales. La evaluación es, pues, una empresa racional, que incluso aspira a legitimarse como científica, pero que a menudo se puede efectuar por pseudorrazones muy poco ciertas.

Conviene estar alerta, pues, y fomentar y generalizar una cultura favorable y cohesionada hacia las bondades y beneficios de la evaluación. Sin actitudes positivas en todas las personas involucradas en la organización, los procesos de cambio y las decisiones que inevitablemente traen consigo las evaluaciones carecerán de utilidad y provecho.

Hasta aquí hemos mencionado razones negativas para evitar la evaluación o para realizarla con fines espurios. Conviene ahora ofrecer algunas de las razones positivas que ayuden al personal de las ONGD a convencerse y convencer de la inaplazable necesidad de instaurar un eficiente ciclo de evaluaciones dentro de su organización. Sin pretensión de exhaustividad ofrecemos las siguientes:

- Razones económicas: por eficacia, la ONGD debe saber si cumple o no con los objetivos que se propone y qué efectos generan sus acciones, tanto deseados como imprevistos, positivos y negativos; por eficiencia, la organización debe conocer -al menos con aproximación razonableel coste de oportunidad de su cartera de proyectos. Seleccionar unos y despreciar otros debe hacerse bajo criterios justificados, donde la eficiencia económica debida a su especialización o por economías de escala, debe tener un peso considerable, aunque no el único debido a su natural ausencia de lucro. La eficacia y eficiencia económicas permiten establecer una visión y estrategia para las acciones con fundamentos no gratuitos.

- Razones éticas: ante todo, por honradez con lo real. En el sistema de valores de la estructura económica actual, las acciones solidarias y las 
donaciones no son evidentes y deben justificarse ante la opinión pública, tanto del Norte como del Sur. La ONGD debe aspirar a ofrecer el mejor producto o servicio posible, a ofrecer lo bueno para sus poblaciones meta, fruto de evaluaciones que prueban esa bondad y nunca "experimentar" con las poblaciones objetivo o beneficiarios.

- Razones pedagógicas: fruto de la inclusión en todo proyecto de un ejercicio evaluador y los procesos de mutuo aprendizaje y capacitación que genera. Toda evaluación es una oportunidad de incrementar el capital humano de todas las partes involucradas. Se producen acciones de empoderamiento, autorresponsabilidad y fortalecimiento comunitario de la parte beneficiada. Se fortalecen las mutuas relaciones entre las partes, se obtienen conclusiones y recomendaciones que impulsan la continuidad de la cooperación. Se aprenden y detectan las claves de los círculos virtuosos y de buenas prácticas, potencialmente reproducibles en otros proyectos, lugares o circunstancias semejantes. Se obtienen materiales e información primaria que pueden ser explotados en las campañas de sensibilización de la opinión pública y en las acciones de presión política. Se examinan conductas y actitudes que pueden hacer crecer la autoestima y la dignidad en las poblaciones empobrecidas y la motivación y satisfacción en el personal de la ONGD, tanto la que actuó como contraparte local, como la financiadora del Norte ${ }^{10}$.

- Razones científicas: en una disciplina que aspira a considerarse actividad investigadora y científica, como es la evaluación, una fuente de legitimación puede ser la evaluación practicada como búsqueda conjunta de la verdad parcial que to dos los implicados en el proyecto de desarrollo tienen. Al ofrecerla en el ejercicio evaluativo, se avanza y se produce conocimiento sobre la pobreza y sus causas, los mecanismos que la consolidan y los que la atenúan, así como los procesos exitosos que solucionan o palian tales situaciones inhumanas. Al poner al ser humano en el centro (a la gente, en expresión frecuentemente utilizada por el PNUD) y buscar la verdad sobre las causas y los remedios contra el empobrecimiento, se está realizando una innegable actividad científica.

- Razones de coherencia: pues no se pueden realizar acciones de cabildeo y presión política sobre las debilidades de un sistema, por ejemplo

10 Algunos de estos efectos se señalan con lucidez en MARSDEN y OAKLeY (1990:4-14). 
el de cooperación pública al desarrollo, y no poder ofrecer ninguna evaluación interna que justifique la coherencia entre el denunciante y lo denunciado. Una denuncia crítica sin alternativas probadas o mínimamente justificables fruto de evaluaciones serias, se convierte en demagogia inútil. Es duro el diagnóstico que sobre algunas ONGs han hecho los pobres en la investigación coordinada por Narayan (2000:6): "Ias personas pobres a veces dan cuenta de que, además de ser rudo y enérgico, el personal de las ONG no sabe escuchar. Sorprendentemente, los pobres dicen que consideran a algunas ONG en gran medida irrelevantes, orientadas al autoservicio, limitadas en su alcance y también corruptas, aunque en grado mucho menor que el Estado". Desde luego la imagen de las ONGD en España es más bien la contraria, la de que son ellas casi las únicas que garantizan que los recursos humanitarios llegan a su destino, y ellas mismas cultivan esa imagen de ser "los buenos de la película" con la crítica sistemática a la cooperación oficial. Pero se rinden pocas cuentas propias y muy generales (no más allá de la distribución geográfica de los proyectos, su volumen y el de los recursos financieros gestionados), pero al no haber evaluaciones no se detallan contenidos concretos sobre los que se posee ventaja comparativa sobre el resto de los actores de la ayuda.

- Razones de conveniencia: por si no bastan las anteriores, de mayor altura, las ONGD pueden considerar que ya están siendo evaluadas sus acciones, no sólo en cada proceso de selección de convocatorias públicas desubvenciones, sino que sus proyectos ya han formado parte del sistema de evaluación de la cooperación española que lleva a cabo la Oficina de Planificación y Evaluación (OPE) ${ }^{11}$. Sirva este "estímulo externo" para animar aún más la instauración de procedimientos internos de evaluación en las ONGD.

\section{Consecuencias para las ONGD derivadas de la implantación de un sistema de evaluación}

Este apartado quiere ofrecer reflexiones sobre las consecuencias que puede tener para una ONGD -una vez decidida con convicción a hacerlo-

11 Nos referimos a la evaluación del programa de 29 proyectos cofinanciados con ONGD españolas llevado a cabo en Haití, República Dominicana y Filipinas, objeto de una evaluación en el año 1999. OPE (2000). 
tratar de instaurar con seriedad y constancia un sistema de evaluación operativo y eficaz. Sólo la ingenuidad o la ignorancia pueden suponer que la tarea de implementar un sistema de evaluación en una organización es tarea sencilla. Para las ONGD, como para el entorno de la cooperación en general, la escasa cultura de evaluación contrasta con su creciente necesidad. Falta ponerla en práctica, con tesón y determinación, sabiendo que los frutos de la evaluación justifican sus costes y esfuerzos, pero que pueden llegar a medio plazo.

Se han estructurado las consecuencias en torno a cuatro aspectos. En primer lugar se exponen las que tendrá la evaluación sobre la identidad de las ONGD. En segundo lugar se comentan los cambios organizativos que supone la tarea de evaluar, tanto en el plan estratégico como en el operacional de la entidad. A continuación se desarrollan las modificaciones presupuestarias que la evaluación lleva consigo y, en último lugar, se describen los cambios sobre los recursos humanos y personal integrante de la ONGD.

\subsection{Cambios sobre la identidad de las ONGD}

Uno de los frutos más valiosos, pero también amenazantes, de la evaluación de sus intervenciones puede darse en el cuestionamiento, crisis, superación y fortalecimiento de la propia identidad organizativa de la ONGD.

Al evaluar, se van teniendo datos más o menos empíricos, sobre la eficacia y eficiencia de las intervenciones que la ONGD diseña o apoya. Conoce el grado de pertinencia de sus proyectos y programas, sus impactos y la viabilidad posterior de sus acciones. Este conjunto de datos debe permitirle entrar en un proceso continuo de reflexión crítica sobre su misión, visión y estrategia.

Decía el latino Prudencio que "para un barco sin rumbo, todos los vientos le son contrarios". Pues bien, el rumbo del desarrollo humano de comunidades y países está sometido a un conjunto numeroso de "vientos" variables que la mayoría de las veces superan toda capacidad de diagnóstico y previsión. La crisis de la política de cooperación para el desarrollo tiene mucho que ver con la escasez de resultados. Las ONGD con más tradición e historia en las tareas de la cooperación conocen bien las limitaciones de sus esfuerzos generosos. Pues precisamente en los momentos de crisis es cuando se hace más urgente la claridad sobre qué problemas se van a afrontar (misión), cómo se va a ver el escenario sobre el que se actúa tras proceder a 
solucionarlos o atenuarlos (visión) y cómo se va a lograr materializar esa visión (estrategia).

Una de las principales dificultades que presenta la misión de las ONGD es el alto nivel de abstracción con que ésta es definida. Las autodefiniciones que aparecen de estas organizaciones en el Directorio de la Coordinadora (CONGDE, 2001) están llenas de lugares comunes: luchar para erradicar la pobreza en el mundo, fortalecer la sociedad civil, promover la solidaridad y el desarrollo de los pueblos. La parte positiva del autoconcepto de muchas ONGD es que se basa en valores, por tanto no se confunde la misión o identidad con una acción o problema concreto. Pero la parte peligrosa es que si los valores no se concretan en actuaciones eficientes, se puede caer en un voluntarismo ingenuo cuando no en acciones que pueden agravar todavía más los conflictos que se desean solucionar ${ }^{12}$.

Fowler (1996) ha denunciado que una de las debilidades de los sistemas actuales que existen para evaluar el rendimiento de las ONGD es la separación asumida entre los proyectos y las características internas de la propia organización, como si tuvieran poco o nada que ver entre sí. Biekart (1999) también se queja de que las escasas evaluaciones de proyectos de ONGD estén centradas en las actuaciones de los beneficiarios y ejecutores y que pocas veces el sujeto de la evaluación sea la propia ONGD del Norte. Propone que la evaluación recoja el rendimiento de la ONGD medido a través de la atención a todos los intereses de los múltiples actores implicados en el proyecto, método denominado auditoría social.

Sanz Corella (1999) propone tres áreas de capacidades para construir un

12 Es pertinente y plástica la expresión "no resolver un lío con otro lío" atribuida a proyectos de ONGD mal diseñados. Gándara sostiene, con razón experimentada, que "las mejores soluciones las da quien está más cerca de las necesidades" apoyando la idea de que las intervenciones de las ONGD deben estar diseñadas en permanente diálogo con los socios beneficiarios o "la contraparte". Véase Gándara (1996). Más radical resulta la denuncia recogida por SAnz CoReLLA (1999:8): "la ayuda puede contribuir a la institucionalización de la violencia -el llamado lado oscuro del humanitarismo- por medio de la liberación de recursos locales que quedan disponibles para perpetuar la guerra, atrayendo el saqueo, facilitando el aislamiento de ciertos grupos o socavando estrategias locales". WAAL (1997) denuncia la afluencia masiva de ayuda a Etiopía tras la campaña de Band-Aid, que contribuyó más a la supervivencia del gobierno etíope, cuyo ejército era la razón principal del hambre, al desviarse la ayuda alimentaria a las milicias gubernamentales y STOREY (1997) realiza una dura crítica contra las actuaciones y descoordinación de las ONGD en el genocidio de Ruanda de 1994. 
marco evaluativo integral que incluya la propia identidad de la ONGD. La organización interna o el ser, que incluye la misión, visión, estrategia y recursos; los vínculos que la organización mantiene con los gobiernos, otras ONGD, contrapartes y beneficiarios, es decir la dimensión de saber relacionarse; y la programación que pueda apreciar el impacto la efectividad de sus acciones, esto es, su hacer. Cuando una organización humanitaria ha reflexionado y sabe dar cuenta propia de su ser, hacer y relaciones, ha ganado la batalla del conocer su identidad y sabrá identificar cuál es el valor añadido que la hace diferente y necesaria en el complejo entramado de la cooperación al desarrollo actual, tan distinto al de la Guerra Fría en la que nació.

En el fondo, mucha de la desconfianza ante las acciones de las ONGD y de las quejas ante la falta de datos sobre sus resultad os están relacionados con su falta de identidad. ¿Para qué sirven organizaciones privadas que utilizan muy predominantemente recursos públicos para financiarse? ¿Quiénes son? ¿Quién está detrás de cada institución? O como gráficamente describe Padrón (1988:188) las ONGD «manejan sumas voluminosas, pero su objetivo no es el lucro. Movilizan la adhesión voluntaria, pero no son iglesias. Insisten en la gratuidad del servicio, pero no son centros de filantropía. Se involucran mucho en política, pero no son partidarias, y, en general, quieren mantenerse a distancia del Estado. Promueven el desarrollo, pero no se especializan en 'productividad'. Realizan investigaciones, pero no son académicas. Su clasificación no resulta fácil. Por esto son comprensibles las sospechas levantadas para con ellas. A menudo se escucha la pregunta desconfiada: 'a fin de cuentas, ia quién sirve esta gente?', formulada incluso por colaboradores próximos».

Esta incertidumbre sobre la propia identidad se acentúa en el contexto de escasez de fondos para la cooperación al desarrollo y de presencia creciente y competitiva de organizaciones locales del Sur, que acuden directamente a las agencias donantes solicitando financiación y prescindiendo del "servicio" de intermediario de la ONGD del Norte. La cadena de ayuda se estrecha al eliminar un eslabón, disminuyen los costes de transacción, aumenta la eficiencia y plantea la pregunta sobre el valor añadido real que aporta la institución del Norte. Muchas ONGD pueden ver en este aspecto un logro, pues puede ser resultado de sus propias acciones de colaboración, fortalecimiento y formación de organizaciones locales que han hecho prescindibles ya sus servicios. Nos encontramos con la paradoja de que, a diferencia de la empresa o las organizaciones lucrativas, el mayor éxito de una organización 
de promoción del desarrollo sea su propia extinción y desaparición, como resultado de haber cumplido su misión.

Otro aspecto relacionado con la identidad de las ONGD es su especialización. Del numeroso abanico de organizaciones españolas, muchas se dedican a lo mismo compitiendo entre sí. Una visión racional y económicamente eficiente del sector apuntaría a procesos de "fusiones" y "especializaciones", en busca de economías de escala o de diferenciación de productos. Los estudios realizados sobre eficiencia comparada entre ONGD españolas ofrecen como criterio principal de valor precisamente el tamaño de la entidad, excluyendo factores como la confesionalidad, ideología o forma jurídica que adopte (Marcuello, 1999).

Nos atrevemos a pronosticar un proceso de alianzas y "fusiones" entre las ONGD españolas a medida que vaya madurando el sector, en búsqueda de la eficiencia y la consolidación de su propia identidad. La homogeneidad de sus actividades es un factor que facilita este proceso y que debe buscar la unión de esfuerzos, la distribución adecuada de las responsabilidades y los aportes, de manera que los recursos y fortalezas de cada ONGD sean aprovechados sumariamente.

\subsection{Cambios organizativos}

Además de cambios en la propia identidad de las ONGD, el sistema de evaluaciones de sus intervenciones tiene consecuencias organizativas para la institución. Entre otros, consideramos los efectos sobre la planificación estratégica de la organización, sobre su plan operacional, y terminaremos esta sección comentando al gunas posibles figuras organizativas que ayuden a un eficaz desarrollo del sistema evaluativo, como una comisión de evaluaciones o un departamento que coordine todas las cuestiones relacionadas con el ciclo de la evaluación.

\section{a) El plan estratégico}

Vernis (1998:71) define este plan como "el esfuerzo disciplinado de producir decisiones y acciones fundamentales que acaben por definir lo que una organización es, lo que hace y por qué lo hace".

Es decir, la organización -sea lucrativa o no- que tiene un plan estratégico sabe dar cuenta de los colectivos preferentes a quienes se dirige, qué 
servicios presta, cómo lo hace y en qué se diferencia de otras organizaciones parecidas $^{13}$.

Un sistema eficiente de evaluación de las intervenciones proporciona a la ONGD, que lo lleva a cabo, elementos en forma de datos y razones (cuantitativos y cualitativos) con los que articular un plan estratégico sobre el que fundamentar sus acciones.

El plan estratégico está en estrecha relación con la misión concreta de la organización (¿quiénes somos y qué sabemos hacer bien?), con el análisis de las oportunidades, amenazas, puntos fuertes y débiles de la organización (su matriz DAFO que le permite responder a ¿dónde estamos y dónde queremos llegar?), y con la insustituible visión que tiene sobre la realidad del sector sobre el que trabaja (¿qué tenemos que hacer para lograr los objetivos que nos proponemos como misión y que justifican nuestra existencia?).

Todos estos interrogantes son muy pertinentes en el momento histórico actual en el que se encuentran las ONGD españolas. Terminado el boom de su creación, se abre la etapa de la profesionalización donde la eficacia y calidad de las intervenciones pesan más que el asistencialismo y las buenas voluntades solas. Los recientes cambios en las condiciones de financiación de los recursos de la Agencia Española de Cooperación Internacional (AECI) han presionado mucho a las ONGD, estableciendo tres niveles: las que reciben financiación ( ¿y confianza?) de largo plazo por medio de las estrategias, las que reciben fondos para sus programas y las que sólo acuden a las convocatorias de proyectos. Dado que muchas de las organizaciones que consiguen fondos privados son precisamente las que copan las estrategias aceptadas en la $\mathrm{AECl}$, a las más pequeñas les está siendo muy difícil competir en este escenario de concentración. Quizá una alternativa es la especialización sectorial y la formación de conciertos y alianzas entre ellas. Pero ¿con quién asociarse? ¿qué criterios deben presidir esta alianza? Precisamente la evaluación puede ofrecer productos en forma de conocimientos que pueden ser utilizados como insumos del plan estratégico, convirtiéndolo en un proceso permanente de reflexión y cambio, dirigido a fortalecer la institución en el presente y delinear su actuar futuro, con el objetivo de que pueda responder de forma adecuada a las condiciones tan rápidamente cambiantes como son las del entorno internacional en materia de cooperación al desarrollo.

13 Sobre la dirección estratégica y control en las ONGD, véanse IríbAR (1996); IRíbAR y MARTínez Pereda (1996). 
Durante la década de los noventa, muchos países han sometido a una profunda reflexión y reforma su sistema gubernamental de ayuda al desarroIlo (Italia, el Reino Unido, Países Bajos o Japón, por ejemplo). También lo han hecho organismos multilaterales como el Banco Mundial. Todos coinciden en que la cooperación no termina de ofrecer resultados satisfactorios y que deben ofrecerse nuevas ideas creativas en este terreno -además de dineroy aumentar los estudios sobre la eficacia de la ayuda. Ya son conocidas las restricciones presupuestarias que los donantes se han ido imponiendo al hilo de la "fatiga de la ayuda". Por otra parte son crecientes las colaboraciones directas entre organismos multilaterales y gobiernos centrales con ONG del Sur. Sin una identidad y estrategia claramente definida y practicada, muchas ONGD se verán marginadas de los procesos realmente significativos de promoción del desarrollo humano y desaparecerán o tendrán crecientes dificultades para autojustificarse.

Por último, el plan estratégico, orientado por los resultados de las evaluaciones, debe aumentar la capacidad de coordinación de la ayuda y de transmisión y circulación de la información sobre las actuaciones llevadas a cabo por las distintas ONGD en lugares comunes o muy cercanos, aspecto éste denunciado como debilidad en la planificación de sus actuaciones.

\section{b) Los cambios operacionales}

Un segundo ámbito de enriquecimiento de los estudios evaluativos es el plan operacional de la organización o su forma de trabajar. Ya hemos hecho referencia a que todos los proyectos formulados y aprobados por la ONGD debe contemplar una forma de evaluación. Es claro que no todos en la misma forma y profundidad, pero que to do proyecto bien formulado, sobre todo si es diseñado bajo el enfoque del Marco Lógico (ML), debe contener un objetivo específico claro, con indicadores mensurables y pertinentes y con un sistema de recogida de información y seguimiento que permita el tipo de evaluación diseñado.

Los proyectos de carácter más económico, productivo o de infraestructu$\mathrm{ra}$, se benefician de su mayor tangibilidad y pueden incidir en indicadores más cuantitativos.

Los proyectos de carácter más social, articularán seguimientos basados en indicadores y herramientas cualitativas que permitan comparaciones de utilidad. 
Los proyectos innovadores, "piloto" o creativos, pueden ser evaluados de forma más detenida y profunda. La evaluación externa o mixta puede incrementar determinados valores, como la independencia o la credibilidad.

La extensión de la cultura de la evaluación, sobre todo en los identificadores y formuladores de proyectos, debe ser una de las tareas más asociadas con los aspectos operacionales de la evaluación.

\section{c) Creación de nuevas figuras organizativas}

Un tercer cambio organizativo que debe producirse para instaurar eficazmente el sistema de evaluación dentro de una ONGD es la creación de alguna figura organizativa que modifique el organigrama de la institución y que le dé operatividad y seguimiento a todo el ciclo de la evaluación.

Esta figura no tiene por qué ser uniforme para todas las ONGD. Dependerá de la propia estructura interna y de su tamaño. Puede ser una comisión o comité de evaluaciones, como se propone en la Metodología Española de Evaluación (MAE 1998), o un departamento de tamaño ajustado para que pueda coordinar todas las acciones de evaluación.

Sus principales funciones serán:

- Velar para que todos los proyectos formulados tengan explicitado el modo en que serán evaluados.

- Asegurarse de que las contrapartes y beneficiarios han participado en el diseño del sistema de evaluación de la intervención y conocen sus responsabilidades y misiones en lo que a evaluación se refiere durante la ejecución y seguimiento de la acción.

- Determinar las evaluaciones más profundas que se llevarán a cabo en cada ejercicio.

- Establecer los términos de referencia de dichas evaluaciones y gestionar to do lo relacionado con las evaluaciones externas o mixtas que se decidan: composición de los equipos, tiempos, presupuesto, objetivos de la evaluación, metodología, etc. en diálogo con el equipo evaluador.

- Sintetizar las recomendaciones de las evaluaciones y componer las distintas colecciones de resultados que vayan ofreciendo las evaluaciones: por países o zonas, por instrumentos de cooperación, temáticas, sectoriales, etc. 
- Organizar la difusión de las evaluaciones, haciendo llegar la información pertinente a los interesados: beneficiarios, formuladores internos, coordinadores de proyectos, directivos, otras ONGD, organismos públicos, investigadores...

- Asegurarse de que las evaluaciones reciben una retroalimentación eficaz y útil.

- Organizar la formación interna y externa del personal de la ONGD en materia de evaluación, a través de seminarios, jornadas de síntesis y formación teórico-práctica en evaluación de proyectos, u otras iniciativas que se consideren relevantes en esta materia.

Los cambios estratégicos y operacionales, así como estas figuras organizativas internas, son tres consecuencias directas de la decisión de evaluar, pero también hay consecuencias presupuestarias y de recursos humanos que pasamos a desarrollar.

\subsection{Consecuencias presupuestarias}

Las decisiones quellegan a concretarse en espacio, tiempo y dinero tienen fuertes garantías de que se llevarán a cabo. De otra manera, pueden resultar bellos deseos, pero nada más. Decidir evaluar es enfrentarse a su coste. Y éste es el factor más abundantemente señalado como razón para no evaluar aún, pero ¿es caro evaluar?

Para ser socialmente solidario, hay que ser económicamente eficiente y autónomo. Sin una gestión eficiente de los recursos (limitados), ninguna organización permanece. Sin grados de autonomía, la dependencia hacia el financiador mina la propia identidad y estrategia de la ONGD. La deseable diversificación financiera de las ONGD españolas y su mayor autonomía es un aspecto que ya ha sido señalado (Larrú, 2001a, 2001b).

En lo referente a la evaluación, creemos que no debe seguirse aplazando la necesidad de evaluar por razones económicas. Con independencia de lo que se crea sobre si evaluar es caro o no, está claro que el coste depende del tipo de evaluación a realizar. Ya es obligatorio presentar informes de seguimiento y finales en todas las intervenciones cofinanciadas, y estas actividades tienen también su coste, que se asume. Las estrategias y programas de la $\mathrm{AECl}$ ya incluyen instrucciones que obligan a presentar una evaluación final.

La propuesta que se hace aquí es evaluar todas las intervenciones de la 
ONGD con independencia de su obligatoriedad externa, aunque cada actividad exige un formato, diseño tiempo y profundidad distinto, pero ya que la debilidad y dependencia financiera es un hecho, el coste debe ser minimizado, pero aprender no es caro. Creemos que un seguimiento funcional, bien determinado por la buena identificación y formulación del proyecto y con unas acciones de seguimiento de calidad, por parte de los responsables ejecutores del proyecto o por personal sobre el terreno, asegura una cantidad de información razonable y barata para hacer una evaluación formativa y de los procesos de desarrollo que aporte mucho valor a la organización. De hecho, la mayoría de los proyectos suelen visitarse sobre el terreno por los responsables de cada país, con lo que el conocimiento ya se genera. Probablemente lo que falta es rentabilizarlo dedicando esfuerzos adicionales a poner los por escrito para que queden al servicio de la organización y no sólo del contratado o directivo. Escribir una pequeña memoria de viaje, útil y centrada en las lecciones aprendidas fruto de lo que se ha detectado, de que funciona bien o mal y por qué, así como los posibles perjudicados por el proyecto y las probabilidades de su sostenibilidad no es caro. Hay que dedicarle tiempo e interés, pero no es caro. Hay que abandonar la idea de que la única manera de evaluar es encargársela a una consultora externa que nos cobrará mucho por este servicio ${ }^{14}$.

Reiteramos aquí la justificación de las evaluaciones como coste de oportunidad de proyectos fracasados, mal diseñados y de los que no se aprende, por no evaluar ni disponer de un sistema de transmisión de buenas y malas prácticas dentro de la ONGD.

El coste de las evaluaciones externas también es opinable. Algunas ONGD ya encargan este tipo de evaluaciones y otras comienzan a estar preparadas para ejecutarlas con personal interno gracias a un proceso formativo específico ${ }^{15}$. Un sistema de concurso y formas creativas de remuneración son campos propicios para la práctica novedosa y creativa que ha caracterizado tradicionalmente a las ONGD.

La financiación de las evaluaciones puede suponer desvío de gasto de unas

${ }^{14}$ A modo de ejemplo, Entreculturas ya ha iniciado un proceso de sistematización escrita de los hallazgos encontrados en los viajes y ha realizado una evaluación mixta de su programa de cobertura educativa en Paraguay. El coste no ha superado apenas los billetes de vuelo de los dos miembros del equipo evaluador.

15 Intermón es un ejemplo del primer caso y Cruz Roja del segundo. 
actividades a otras en función de la prioridad que se otorgue a cada una ${ }^{16}$. 0 bien, generar nuevas fuentes de ingreso para dedicarlos a la evaluación.

Ya hemos comentado la posibilidad de introducir el coste de la evaluación como coste indirecto en la cofinanciación española de proyectos, aunque esto vaya en detrimento de los costes de identificación, administración, personal y otros, a los que ahora se destina.

En definitiva, la voluntad de evaluar se expresa en prioridades, donde el coste es un elemento más a tener en cuenta, pero nunca debe ser la razón acomodaticia para resistirse a su ejecución e implantación. Las múltiples razones ofrecidas en este trabajo refuerzan la necesidad de financiar de forma responsable esta actividad, por otra parte contemplada en la Gestión del Ciclo del Proyecto que dicen utilizar la mayoría delas organizaciones. Hay que llevar el proceso circular hasta el final y dejar que la evaluación aporte su valor.

\subsection{Consecuencias sobre los recursos humanos}

La creciente profesionalización que acontece dentro de algunas ONGD, tanto por la creciente complejidad de sus trabajos, como por la necesidad de contar con un personal estable y remunerado que dé continuidad a las principales acciones gestoras de la organización, es un hecho innegable $e^{17}$.

El equilibrio entre profesionales y voluntarios no es sencillo. Si los primeros aportan rigor y continuidad en el trabajo, asumiendo responsabilidades y tomando decisiones, los voluntarios son la legitimación de la identidad de la ONGD como organización de la sociedad civil, ahorran costes y ofrecen su experiencia y solidaridad en los espacios de tiempo que colaboran con la entidad. Su trabajo puede ser tan riguroso o más que el de los profesionales, pero no suelen asumir tantas decisiones ni responsabilidades y, salvo excepciones, no prestan un trabajo continuado y estable sobre el que gestionar el día a día en la organización ${ }^{18}$.

16 BIEKART (1999) hace una provocativa comparación entre el coste de campañas publicitarias para la recaudación de fondos de algunas ONGD y la excusa del alto coste de la evaluación.

17 Una profundización deaspectos dela profesionalización del personal delas ONGD seencuentra en Linard (1994a, 1994b); Vicente Cruz (1995,1996); Estébanez y Alonso (1996); Serrano (2001).

18 Una figura “intermedia” de gran interés y rendimiento son los voluntarios a tiempo completo en la organización. Normalmente jubilados que prestan su experiencia profesional laboral, ahora al servicio de los más necesitados a través de la ONGD. 
Como ha señalado Sanz Corella (1999:21) "a medida que las ONGs se hacen más profesionales, la escala de las operaciones es mayor, se manejan presupuestos millonarios y las estructuras de apoyo y burocracias son más pesadas, es cada vez más difícil resistir a las presiones que amenazan con erosionar seriamente sus características distintivas más valiosas; es decir, sus puntos fuertes o 'ventajas comparativas'" 19 .

El equilibrio que salvaguarde estos peligros y mantenga las ventajas de las dos figuras, profesionales y voluntarios, lo intenta describir Vernis bajo la denominación de "profesionales voluntarios y voluntarios profesionales". "Los profesionales voluntarios son las personas que se dedican de forma remunerada a trabajar en una organización no lucrativa... Un profesional voluntario está motivado por la misión de la organización, trata de entender a las personas voluntarias que trabajan en ella, intenta ser constructivo... Al mismo tiempo, un profesional voluntario sabe que continuamente ha de estar aprendiendo. En otras palabras, para trabajar en una organización no lucrativa hay que ser no sólo un excelente profesional, sino estar imbuido de los valores de la organización" (Vernis, 1998:152).

De la misma manera, el solo hecho de ser voluntario no se debe traducir en valor añadido de escasa calidad, sino que su trabajo debe tender a ser lo más "profesional" posible.

Es indudable que la evaluación exige una cualificación amplia y que los evaluadores, en la mayoría de los casos, deben ser profesionales. Ninguna ONGD lleva a cabo evaluaciones sólo con voluntarios. Cuando intervienen miembros internos son especialistas en proyectos o lazona geográfica donde se llevó a cabo la intervención es la suya o es un amplio conocedor del instrumento utilizado en el proyecto.

Está clara la relación entre coste de la evaluación, el tiempo necesario para realizarla y la cualificación requerida al personal evaluador. Por su complejidad, si se desea incorporar a personal interno a las evaluaciones, deberán recibir abundante formación técnica específica. Creemos que puede formarse a voluntarios profesionales o a profesionales internos de la organización para formar parte de evaluaciones mixtas o para realizar evaluaciones simples, aquellas exigibles a todos los proyectos. Para las evaluaciones en

19 Los puntos fuertes que señala son: el compromiso, su carácter, la flexibilidad, el cuidado, la comprensión cercana de la cultura y ambientes locales y la espontaneidad. 
profundidad, es más deseable que el equipo sea mixto o externo y completamente profesional.

La formación inicial a to do el equipo del departamento de proyectos de la ONGD tiene una sinergia con la formación que éstos pueden dar al personal de la contraparte local y a los beneficiarios, sobre todo a los miembros encargados de recopilar la información necesaria para la elaboración de los indicadores de seguimiento y evaluación final.

Además de la necesidad de formación, hay otros dos aspectos vinculados a la evaluación y el capital humano de la organización: la rotación del personal y el nivel salarial.

Respecto a los salarios de los profesionales de las ONGD, no es éste el lugar para su debate. Pero sí se desea mencionar -por su relación con la evaluación- el que un nivel salarial "justo" tiene como ventaja el que el personal cualificado y experimentado, permanezca en la organización.

Uno de los posibles motivos por los que la rotación del personal profesional sea tan elevada en las ONGD, puede ser la incapacidad de la organización de retener sus servicios y activos con un salario suficientemente atractivo. Al estar en una organización no lucrativa, los costes no asociados a las acciones directas de desarrollo se consideran de menor valor añadido 20 . Pero el problema para la evaluación es importante, pues la rotación continua del personal supone la no acumulación de las experiencias personales, técnicas y profesionales. Por tanto, las causas de los éxitos y fracasos de las intervenciones puntuales y de los procesos de desarrollo se pierden. Este fenómeno se acentúa cuando no hay evaluaciones escritas que vayan recogiendo las enseñanzas y buenas prácticas acumuladas por la organización. La ONGD no se convierte en una organización de aprendizaje. Denuncia, pero no es capaz de aportar soluciones creativas basadas en la generación de su propio conocimiento original. Las campañas se alimentan de datos, ideas y medios externos, a veces poco internalizados.

En resumen, evaluar las acciones tiene, al menos, tres consecuencias sobre los recursos humanos de una ONGD: requiere alta cualificación en su personal (conocimientos generales en todo el staff de proyectos y conocimientos especiales en los miembros de la comisión o departamento de

20 Piénsese en las pocas ONGD que tienen un departamento de estudios de calidad, con personal remunerado. 
evaluaciones); requiere, en segundo lugar, remuneraciones eficientes y formación permanente de los profesionales y, en tercer lugar, requiere estabilidad de su personal, al menos a medio plazo. Los tres aspectos están estrechamente interrelacionados. Incluso puede contemplarse la formación interna como una "remuneración en especie", complementaria al salario, pues es uno de los aspectos más valorados por el personal interno de las ONGD, a priori más motivado en su labor y que renuncia a los salarios del mercado, si a cambio obtiene otras contraprestaciones y gratificaciones personales no dinerarias. La empleabilidad de los profesionales de las ONGD puede depender más de la formación y los aspectos intangibles de su contraprestación que del nivel salarial ${ }^{21}$.

Con un personal estable, cualificado y motivado, el ciclo y la cultura de la evaluación tienen más garantías de irse implantando con éxito y ofreciendo los resultados demandados. Con personal formado, tanto en sede como sobre el terreno, su participación en ejercicios de evaluación puede ir creciendo y esto aminora su coste. Los aprendizajes se irán acumulando y las colecciones de síntesis de evaluaciones desplegarán todos sus activos potenciales. Las evaluaciones serán realmente útiles y prácticas y el ciclo de la evaluación se afianzará con fuerza en la cultura y know how de la organización.

\section{Conclusión}

El primer paso que deben dar las ONGD para instaurar internamente un ciclo de evaluaciones eficaz, es convencerse de su necesidad y decidir implantarlo.

Entre las muchas razones para evaluar las acciones de las ONGD, hemos destacado razones económicas (eficacia y eficiencia), éticas (honradez, oferta de proyectos de probada bondad), pedagógicas (aprender de forma permanente, capacitar y "empoderar" a los beneficiarios a través de la evaluación, fortalecer las mutuas relaciones entre las partes que intervienen), científicas (búsqueda de la verdad sobre la pobreza, sus causas y

${ }^{21}$ En una entrevista mantenida con el director de la Fundación Codespa, me comentó que un estudio encargado por su organización a una consultora externa, acerca de la valoración que hacía su personal sobre los componentes de las retribuciones, señaló que -en un nivel suficiente de salario- el factor más apreciado y demandado era precisamente una formación y cualificación que permitiera su empleabilidad. 
mecanismos atenuadores), de coherencia (no se debe criticar lo ajeno sin evaluar lo propio), o de conveniencia (prepararse para evaluaciones externas por parte de organismos públicos).

La evaluación se realiza para mejorar, es decir, exige cambiar y, por tanto, querer cambiar. Las principales consecuencias de la implantación de un sistema de evaluaciones en una ONGD afectan a su propia identidad, a su organización, a sus presupuestos y a sus recursos humanos.

Las evaluaciones pueden ayudar a fortalecer la identidad de las ONGD así como impulsar alianzas y cooperaciones más estrechas entre ellas. A raíz de la reflexión sobre los resultados de las evaluaciones, las ONGD de vocación más generalista pueden buscar concentraciones verticales en busca de economías de alcance que aumenten su eficiencia. Por el contrario, las ONGD más especialistas pueden buscar concentraciones horizontales que les permitan obtener economías de escala.

El principal cambio organizativo que afectará a las ONGD que implanten un sistema de evaluaciones será el de crear una comisión de evaluaciones con las funciones ya expuestas.

Por lo que respecta a los presupuestos, habrá que contar con los costes de la evaluaciones. Consideradas las evaluaciones como una inversión y no como un gasto, y contemplando los ahorros que supone ejecutar proyectos cada vez más exitosos, consideramos que el coste asociado a las evaluaciones debe ser perfectamente asumible por las ONGD, adaptando esta carga presupuestaria adicional a su situación específica y de forma paulatina.

Por último, en cuanto a los recursos humanos se refiere, las evaluaciones exigirán personal especializado, acciones formativas y unas remuneraciones de eficiencia que les permita contar con profesionales voluntarios, motivados y excelentes. 


\section{Bibliografía}

BIEKART, K. (1999), “Evaluación del impacto de la ayuda privada orientada al fortalecimiento de la sociedad civil", Alonso, J.A. y Mostey, P. (eds.), La eficacia de la cooperación internacional al desarrollo: evaluación de la ayuda, Madrid, Civitas. Pp. 185-238.

CAD (2002), Spain. Development Co-operation Review, Paris, Development Assistance Committee. OECD.

Carvalho, S. y White, H. (1997), "Combining the Quantitative and Qualitative Approaches to Poverty Measurement and Analysis: The Practice and the Potential", Washington. Technical Paper 366. World Bank.

Chambers, R. (1997), Whose Reality Counts? Putting the first, last, London, Intermediate Technology Publications.

CONGDE (2001), Directorio de ONGD 2000, Madrid, Coordinadora de ONGD de España.

EDWARDS, M. (1997), "Organizational Learning in Non-governmental Organizations. What have we learned?", Public Administration and Development, vol. 17, 235-250.

Edwards, M. y HuLme, D. (1996) (1998²), Beyond the Magic Bullet: NGO Performance and Accountability in the Post-Cold War World, London, Earthscan Publications - Save the Children Fund.

Estébanez, P.y Alonso, B. (1996), “EI Voluntariado, un reto para la Organización de la acción humanitaria”, Boletín de Estudios Económicos 158, 299-310.

FowLER, A. (1996), “Assessing NGO performance: difficulties, dilemmas and a way ahead", EdwARDs, M. y HULME, D., Beyond the magic bullet: NGO Performance and Accountability in the Post-cold War World, London, Earthscan Publications - Save the Children Fund.

GándARA, J. (1996), Las empresas de desarrollo, Guatemala, FUNDAP. Mimeo.

García Ramos, J. M. (1992), "Recursos metodológicos en la evaluación de programas", Bordón 43-4, 461-476.

GonZález, L. (2000), "La evaluación expost o de impacto. Un reto para la gestión de proyectos de cooperación internacional al desarrollo", Bilbao, Cuadernos de Trabajo de HEGOA, no 29. 
GuBA, E. G. y LinCoLn, Y. S. (1989), Fourth Generation Evaluation, Newbury Park, Sage.

IRíbAR, M. F. (1996), "Las Organizaciones de Ayuda Humanitaria y la dirección Estratégica", Capital Humano 85, 22-28.

Iríbar, M. F. y Martínez Pereda, J. (1996), “CCómo podemos controlar la gestión desarrollada por nuestra ONG?", Boletín de Estudios Económicos 158, 245-260.

IUDC-UCM (1999), Revista Española de Desarrollo y Cooperación ㄲo 4.

LARRú, J. M. (2000), La evaluación en los proyectos de cooperación al desarrollo de las ONGD españolas, Madrid, Universidad San Pablo-CEU. Tesis Doctoral.

- (2001a), "Análisis de los proyectos e ingresos de las ONGD de la CONGDE en 1999", en Directorio de ONGD 2000, Madrid, Coordinadora de ONGD de España. Pp. 13-30.

- (2001b), “¿Públicas o privadas? La financiación de las ONGD españolas", Revista de Fomento Social 56 (221), 93-127.

LINARD, A. (1994a), "Rehabilitar el desarrollo. Pero ¿qué desarrollo?", Revista de Fomento Social 49, 351-371.

- (1994b), "Seis desafíos para la cooperación al desarrollo", Revista de Fomento Social 49, 495-515.

MAE (1998), Metodología de Evaluación de la Cooperación Española, Madrid, MAE-SECIPI.

- (2001), Metodología de Evaluación de la Cooperación Española II, Madrid, MAE-SECIPI.

Marcuello, C. (1999), "Análisis de la conducta y eficiencia de las ONGD españolas", Información Comercial Española 778, 181-196.

M ARSDEN, D. y OAKLEY, P. (eds.), (1990), Evaluating social development projects, Oxford, Oxfam.

Narayan, D. (2000), La Voz de los Pobres. ¿Hay alguien que nos escuche? MundiPrensa, Madrid, http://www.worldbank.org/poverty/spanish/voices/ voll.htm

OPE (2000) Programa de subvenciones y ayudas a ONGD en Haití, República 
Dominicana y Filipinas, Madrid, Informes de evaluación 8/2000. MAESECIPI.

PADRón, M. (ed.), (1988), Las Organizaciones No Gubernamentales de DesarroIlo en el Perú, Lima, Centro de Estudios y Promoción del Desarrollo.

Patton, M. Q. (1997), Utilization-Focused Evaluation. The New Century Text, Thousand Oaks, Sage.

RIDDELL, R. (1990), "J udging Success. Evaluating NGO Approaches to Alleviating Poverty in Developing Countries", London, Overseas Development Institute. Working Paper 37.

- (1998), Evaluating NGO Development Interventions. Documento de trabajo del Seminario: Alonso, J. A. y Mosley, P. (Dtores.), "La eficacia de la cooperación internacional al desarrollo: evaluación dela ayuda", Santander, UIMP. [Traducido y publicado en Alonso y MosLey (1999) pp. 239-265].

Rовв, C. (1999), "Can the Poor Influence Poverty? Participatory Poverty Assessments in the Developing World", Washington D. C., World Bank.

Rossi, P. H. y Freeman, H. E. (1989), Evaluation. A Systematic Approach, Newbury Park, Sage.

Salmen, L. (1995), "Participatory Poverty Assessment: Incorporating Poor People's Perspectives into Poverty Assessment Work", Washington, Social Development Paper, no 11 . World Bank.

- (1998), "Toward a Listening Bank: A Review of Best Practices and the Efficacy of Beneficiary Assessment", Washington. Social DevelopmentPaper, no 23, World Bank.

Sanz Corella, B. (1999), "Las ONGs en los conflictos internacionales: crisis de identidad y fortaleza institucional". Working Paper, no 8. Instituto Internacional de Gobernabilidad. http://www.iigov.org/pnud/bibliote/paper/ paper $8 / \mathrm{htm}$.

Serrano, M. (2001), "Las ONGs entre la empresa y el Estado: ¿cambio o reproducción del sistema?" en NiETo, L. (coord.), "Cooperación para el desarrollo y ONG. Una visión crítica", Madrid, La Catarata-IUDC, pp. 141-169.

Storey, A. (1997), "Non neutral humanitarism: NGOs and the Rwanda crisis", Development in Practice 7 (4), 384-394. 
TEJADA, L. (2002), "El examen del CAD y la cooperación española", Revista Española de Desarrollo y Cooperación 10, 115-131.

VernIs, A. et al. (1998), La Gestión de las Organizaciones No Lucrativas, Bilbao, Deusto.

Vicente Cruz, E. (1995), "La gestión de los Recursos Humanos en las organizaciones de ayuda humanitaria", Capital Humano 81, 10-20.

- (1996), "La entrevista de selección de voluntarios y colaboradores. Características específicas y aspectos prácticos", Boletín de Estudios Económi$\cos 158,277-298$.

WaAL, A de (1997), Famine Crimes: Politics and the disaster relief industry in Africa, London, J ames Currey.

Weiss, C. H. (1972) (1998²), Evaluation Research, New Jersey, Prentice-Hall. Englewood Cliffs. [Hay traducción castellana en Trillas, México (1975 y 1992)].

WHITE, H. (2002), "Combining Quantitative and Qualitative Approaches in Poverty Analysis", World Development 30 (3), 511-522. 